\title{
Approche diagnostique et thérapeutique des douleurs neuropathiques trigéminales post-traumatiques : à propos de 10 cas.
}

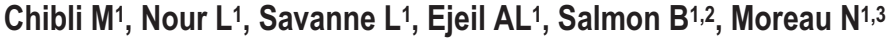 \\ 1. Service d'Odontologie, Hôpital Bretonneau - AP-HP - Paris \\ 2. Pathologie, Imagerie et Biothérapies Orofaciales, UFR Odontologie - Université Paris V - Paris Descartes \\ 3. Centre de Psychiatrie et Neurosciences, Inserm : U894, Université Paris V - Paris Descartes
}

\section{Introduction}

Les Douleurs Neuropathiques Trigéminales Post-Traumatiques (DNTPT) définissent un sous-type particulier de neuropathies trigéminales, résultant d'un trauma physique ou chirurgical de la région oro-faciale, se présentant sous la forme de signes cliniques positifs (douleur spontanée, hyperalgésie, allodynie) ou négatifs (hypoesthésie) dans le territoire du nerf lésé (Benoliel et al., 2012 a b).

Ces neuropathies représenteraient une complication iatrogène de 0,5 à $12 \%$ des actes de chirurgie orale, incluant les traitements endodontiques, la chirurgie apicale, les avulsions dentaires, les énucléations kystiques, la chirurgie orthognathique ou la chirurgie implantaire. (Benoliel et al., 2012 a b)

Malgré des caractéristiques communes aux autres douleurs neuropathiques, les DNTPT présentent des spécificités physiopathologiques et thérapeutiques qui rendent leur diagnostic et leur prise en charge particulièrement complexes, se traduisant le plus souvent par une errance diagnostique majeure.

\section{Observation}

II est rapporté une série de 10 cas de DNTPT, diagnostiqués au sein de la consultation de douleurs chroniques oro-faciales de l'hôpital Bretonneau à Paris.

Cette série de cas était composée exclusivement de femmes, âgées de 16 à 65 ans, dont le diagnostic de DNTPT a été posé d'après les critères de Benoliel (Benoliel et al., 2012 b). Sur ces 10 cas, 4 cas faisaient suite à une avulsion dentaire, 3 cas à un traitement endodontique, 2 cas à une chirurgie apicale et 1 cas à une ostéotomie bi-maxillaire à visée orthognathique.

Différents traitements ont été proposés selon l'étiologie, la réponse au traitement et la tolérance individuelle du patient, incluant des anticonvulsivants (prégabaline, clonazépam), des antidépresseurs (amitriptyline) ou des alcaloïdes topiques (capsaïcine). La réponse au traitement a été évaluée sur le plan qualitatif (amélioration de la qualité de vie et du sommeil) et quantitatif (intensité douloureuse mesurée sur une échelle visuelle analogique).

Hormis une patiente ayant refusé tout traitement, plus de $80 \%$ des patients traités ont eu une réponse partielle (diminution de l'intensité douloureuse supérieure à $50 \%$ sur l'EVA) ou totale (disparition complète des douleurs) à leur traitement.

\section{Discussion}

Cette série de cas illustre la diversité étiologique et thérapeutique des cas de DNTPT observables dans nos consultations. La complexité de la prise en charge de ces pathologies douloureuses réside dans :

la difficulté du diagnostic positif et étiologique de la DNTPT,

l'absence de recommandations de prise en charge pour ces neuropathies, une réponse individuelle au traitement souvent imprévisible.

Afin de faciliter le diagnostic et la prise en charge de ces pathologies complexes extrêmement invalidantes, nous proposons un arbre décisionnel de prise en charge de ces pathologies, basé sur cette série de cas, les données de la littérature scientifique et les recommandations scientifiques de prise en charge des douleurs neuropathiques non-trigéminales (EFNS 2010 ; Attal et al., 2010).

(C) The authors, published by EDP Sciences. This is an Open Access article distributed under the terms of the Creative Commons Attribution License 4.0 (http://creativecommons.org/licenses/by/4.0/). 
$65^{\text {ème }}$ Congrès de la SFCO

\section{Conclusion}

Les DNTPT sont des complications post-chirurgicales relativement peu fréquentes mais dont le retentissement fonctionnel et l'impact majeur sur la qualité de vie doivent inciter à un diagnostic et une prise en charge précoces de la part du chirurgien oral.

mehdi.chibli@hotmail.fr 